\title{
Registration Algorithms for Interventional MRI-guided Treatment of the Prostate
}

\author{
Baowei Fei $^{\text {a }}$, Kristin Frinkley ${ }^{a}$, David L Wilson ${ }^{\text {a }}{ }^{* *}$ \\ ${ }^{a}$ Department of Biomedical Engineering, Case Western Reserve University, OH 44106 \\ ${ }^{\mathrm{b}}$ Department of Radiology, University Hospitals of Cleveland, OH 44106
}

\begin{abstract}
We are investigating interventional MRI (iMRI) guided radiofrequency thermal ablation for the minimally invasive treatment of prostate cancer. Nuclear medicine and MR spectroscopy can detect and localize tumor in the prostate not reliably seen in MR. We are investigating methods to combine the advantages of functional images such as SPECT with iMRI-guided treatments. Our concept is to first register the low-resolution functional images with a high resolution MR volume. Then by registering the high-resolution MR image with live-time iMRI acquisitions, we can, in turn, map the functional data and high-resolution anatomic information to iMRI images for improved tumor targeting. In this study, we registered noisy, thick iMRI image slices with high-resolution MR volumes and called this slice-to-volume registration. We investigated two similarity measures, i.e., mutual information and correlation coefficient, and three interpolation methods, i.e., tri-linear, re-normalized sinc, and nearest neighbor. To assess the quality of registration, we calculated 3D displacement on a voxel-by-voxel basis over a volume of interest (VOI) between slice-to-volume registration and volume-to-volume registration that was previously shown to be quite accurate for these image pairs. Over 300 registration experiments showed that transverse slice images covering the prostate work best with a registration error of only $0.4 \pm 0.2 \mathrm{~mm}$. Error was greater at other slice orientations and positions. Since live-time iMRI images are used for guidance and registered images are used for adjunctive information, the accuracy and robustness of slice-to-volume registration is very probably adequate.
\end{abstract}

Keywords: Image registration, medical imaging, interventional magnetic resonance imaging (iMRI), mutual information, thermal ablation, prostate cancer.

\section{INTRODUCTION}

We are investigating interventional MRI (iMRI) guided radiofrequency (RF) thermal ablation for the minimally invasive treatment of prostate cancer. Nuclear medicine and MR spectroscopy can detect and localize tumor in the prostate not reliably seen in MR. We are investigating methods to combine the advantages of functional images such as SPECT with iMRI-guided treatments. Our concept is to first register the low-resolution functional images with a high resolution MRI. Then by registering the high-resolution MR volume with live-time iMRI acquisitions, we can, in turn, map the functional data and high-resolution anatomic information to iMRI images to aid tumor targeting.

Previous success with registering one MR prostate volume to another ${ }^{1}$ encourages us to pursue this plan. We call this volume-to-volume registration, or VV. We used a rigid body, mutual information registration method with some features to improve robustness. ${ }^{1}$ We carefully evaluated registration quality using a variety of methods. For volume pairs acquired over a short time span from a supine subject with legs flat on the table, registration accuracy of both

* BXF18@po.cwru.edu, ** DLW@po.cwru.edu, Wickenden Building 319, 10900 Euclid Avenue, Cleveland, OH 44106.

Visualization, Display, and Image-Guided Procedures, Robert L. Galloway, Editor, 
prostate centroids (typically $<1 \mathrm{~mm}$ ) and bony landmarks (average $1.6 \mathrm{~mm}$ ) was on the order of a voxel $(\approx 1.4 \mathrm{~mm})$. For volumes acquired under very different conditions, e.g., legs flat and legs raised into the treatment position, or with and without bladder or rectal filling, we obtained somewhat larger prostate centroids registration errors of about 3.0 $\mathrm{mm}$.

In this study, we are investigating methods to register live-time iMRI image slices with a previously obtained, high resolution MRI volume. We call this slice-to-volume (SV) registration. Because of our success with VV prostate registration, we can determine SV accuracy by comparing results to VV registration for volume pairs having low VV registration error.

The application of SV registration methods to iMRI-guided treatment of prostate cancer raises several challenges. First, iMRI images often have lower signal to noise ratio (SNR) than diagnostic MR images because of the emphasis on fast imaging and because of the typically lower field strength of open iMRI magnets. Second, a single slice, or a few slices, provides many fewer structures than an entire volume for voxel based matching. Third, the prostate can move relative to the pelvic bones due to changes in rectal and bladder filling ${ }^{2,3}$ or changes in patient posture for treatment. ${ }^{1}$ That is, alignment of the pelvic bones, prominent anatomical features in MR gray-scale images, does not necessarily ensure that the prostate is aligned. Fourth, the normal prostate is a small organ; when healthy, it measures only $\approx 3.8 \mathrm{~cm}$ in its widest dimension. ${ }^{4}$ The small prostate is located below the much larger bladder that can change its shape and size during imaging. Finally, times for registration and algorithm robustness are of particular concern for this application.

Previously reported methods for SV registration were mainly applied to the brain for applications of functional MRI, ${ }^{5,6}$ postmortem pathology studies, ${ }^{7}$ and anatomical modeling. ${ }^{8}$

We previously reported an SV registration method for the application of iMRI-guided treatment of the prostate. ${ }^{9-12}$ In this report, we further investigated the algorithms by comparing three interpolation methods and two similarity measures. We registered noisy, thick iMRI image slices with high-resolution MR volumes. Over 300 registration experiments were performed with 9 pairs of MR volume images acquired from three health volunteers.

\section{REGISTRATION ALGORITHM}

\subsection{Similarity Measurements}

We used two similarity measures, mutual information and correlation coefficient (CC), in our registration. One image $R$ is the reference, and the other $F$ is floating. Their mutual information $\operatorname{MI}(R, F)$ is given below. ${ }^{13}$

$$
M I(R, F)=\sum_{r, f} p_{R F}(r, f) \log \frac{p_{R F}(r, f)}{p_{R}(r) \cdot p_{F}(f)}
$$

The joint probability $p_{R F}(r, f)$ and the marginal probabilities $p_{R}(r)$ of the reference image and $p_{F}(f)$ of the floating image, can be estimated from the normalized joint intensity histograms. When two images are geometrically aligned, MI is maximal. ${ }^{13}$ The correlation coefficient $C C(R, F)$ is given below. ${ }^{14}$

$$
C C(R, F)=\frac{\sum(R(r)-\bar{R}(r))(F(f)-\bar{F}(f))}{\sqrt{\sum(R(r)-\bar{R}(r))^{2} \sum(F(f)-\bar{F}(f))^{2}}}
$$

Here $\bar{R}(r), \bar{F}(f)$ denote the average intensities of the reference and floating images and the summation includes all voxels within the overlap of both images.

Visualization, Display, and Image-Guided Procedures, Robert L. Galloway, Editor, 


\subsection{Interpolation Methods}

We used three interpolation methods, i.e., nearest neighbor (NN), tri-linear, and re-normalized sinc interpolation. ${ }^{15,16}$ The conventional sinc interpolation with a cosine Hamming window is described as below. ${ }^{15}$

$$
\begin{gathered}
I^{\prime}(x, y, z)=\sum_{X} \sum_{Y} \sum_{Z} I(X, Y, Z) \cdot H S(x, X, R) \cdot H S(y, Y, R) \cdot H S(z, Z, R) \\
H S(\alpha, A, R)=\frac{\sin [\pi(\alpha-A)]}{2 \pi(\alpha-A)} \cdot\{1+\cos [\pi(\alpha-A) / R+1]\},
\end{gathered}
$$

with

where $X, Y, Z$, are the coordinates of the original data set $(I) ; x, y, z$ are the coordinates of the re-formatting data set $\left(I^{\prime}\right)$; $A$ is a symbol representing $X, Y$, or $Z$, and $a$ represents $x, y$, or $z$; and $R$ is the kernel size. The Hamming function eliminates problems with oscillatory effects at discontinuities and guarantees that the convolution coefficients fall of to zero at the edge of the sinc kernel (i.e., at $|a|=R+1$, where $R=5$ in this study). ${ }^{15}$

A previous report ${ }^{15}$ showed that re-normalizing the interpolation kernel for a constant integral could make significant improvement in performance of the conventional sinc interpolation. In our implementation, we used the renormalized sinc interpolation method. ${ }^{15}$ We replaced $H S$ in the above equation with

$$
H S^{\prime}(\alpha, A, R)=H S(\alpha, A, R) / \sum_{A} H S(\alpha, A, R)=H S(\alpha, A, R) /|H S(\alpha)| .
$$

\subsection{Comparison of Different Similarity Measures and Interpolation Methods}

We compared two similarity measures, CC and MI, at different resolutions in order to determine their suitability for SV registration. At $1 / 4$ resolution, we resampled images so as to give $1 / 4$ number of the voxels along each linear dimension. At full resolution, we used the full number of voxels. We plot the two similarity measures as a function of translations. After two typical high-resolution MR volumes were registered, ${ }^{1}$ values were plotted with the origin as the optimal transformation. We calculated CC and MI values while moving the simulated iMRI image relative to the highresolution MR image along coronal (anterior-posterior) axis. When obtaining floating images, we used the three different interpolation methods.

Features of MI and CC demonstrate their suitability at high and low resolutions, respectively. At 1/4 resolution, CC curves are much smoother than MI, which is noisy and contains many local maxima as shown in Figures 1. At full resolution, Figures 2 shows that MI curves are much peaked than $\mathrm{CC}$, but once again there is high frequency noise in the MI curves, far from the optimum, that gives rise to local maxima that must be avoided. From these figures, we infer that $\mathrm{CC}$ is better at low resolution and $\mathrm{MI}$ is better at full resolution, when one is close to the optimum value.

We also compared the effects interpolation methods. At 1/4 resolution (Figure 1), the MI curves with sinc had more local maximums than those with tri-linear; however, CC plots with sinc were smoother than tri-linear. This indicates that $\mathrm{CC}$ and sinc are possibly the best choices for robust registration at low resolution. At full resolution (Figure 2), MI and sinc gave the sharpest peak at the optimum; nearest neighbor interpolation gave a flat peak with a width of one voxel; and tri-linear gave a result between the other two. These results imply that MI and sinc interpolation should provide the best registration accuracy at full resolution. However, tri-linear interpolation is about 100 times faster than sinc interpolation in our implementations. 

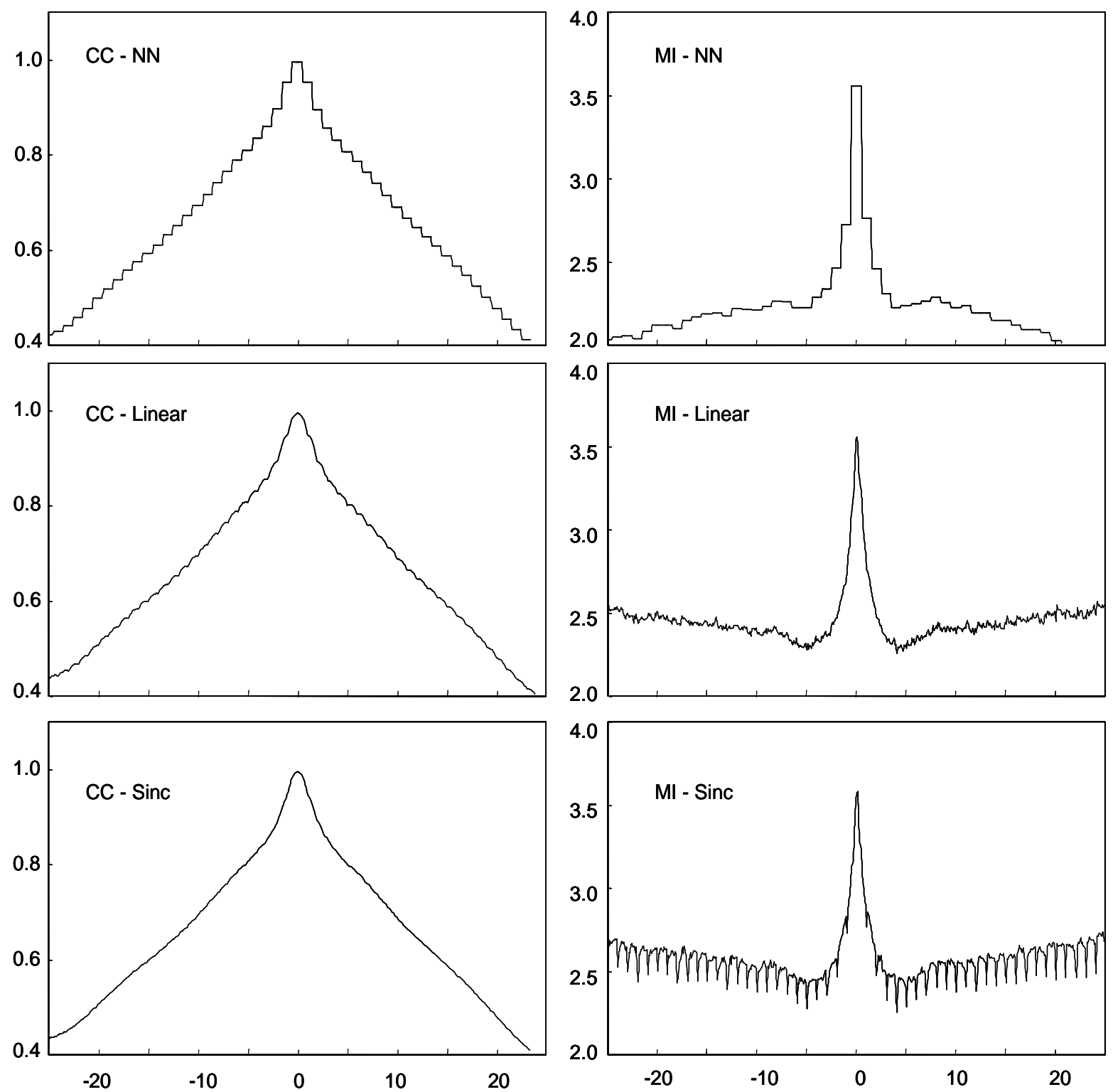

Figure 1. Similarity functions are plotted as a function of translations at the $1 / 4$ resolution in the multi-resolution registration process. Two high-resolution MRI volumes were registered, and they are down sampled by $1 / 4$ along each linear dimension, giving a distance between voxel centers of $\approx 5.5 \mathrm{~mm}$. From the optimal parameters, we computed the similarity of the simulated iMRI and MRI images as a function of translations along the coronal (anterior-posterior) axis. Correlation coefficient (CC) is plotted in the left column. Mutual information (MI) is plotted in the right column. From the top to bottom, nearest neighbor (NN), tri-linear and re-normalized sinc interpolation methods are used to obtain the floating images, respectively. The noisey MI curves in the right column show many local maxima, especially with sinc interpolation. CC curves in the left column are much smoother indicating its suitability for low resolution. Nearest neighbor has a flat peak with a width of one voxel in both similarity curves. The unit of the $\mathrm{X}$-axis is voxel. Images are from volunteer $\mathrm{S} 2$. 

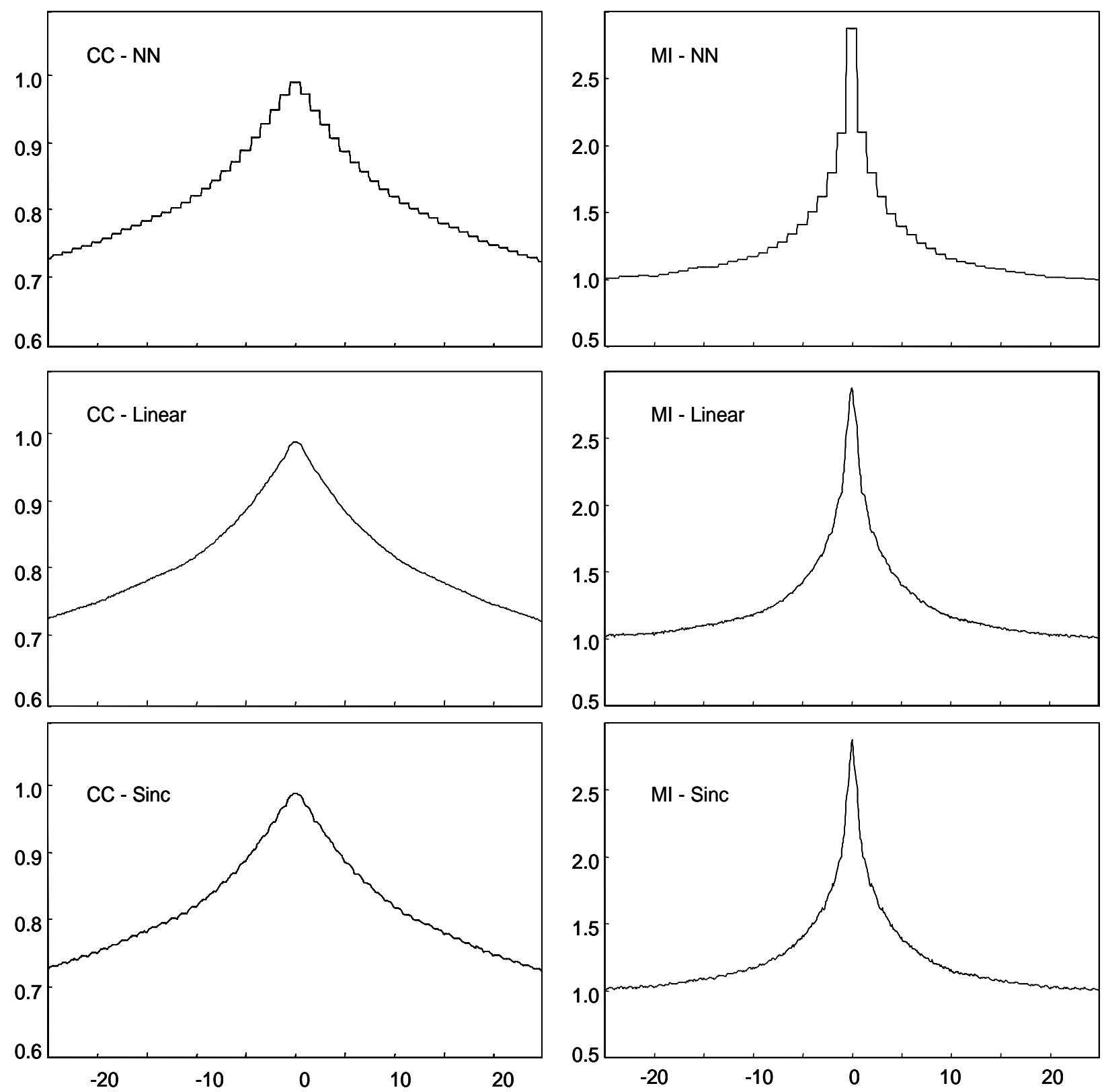

Figure 2. Similarity functions are plotted as a function of translations at full resolution. Many details are given in the legend of Figure 1. Again, CC is plotted in the left column. MI is plotted in the right column. From the top to bottom, nearest neighbor (NN), tri-linear, and re-normalized sinc interpolation methods are used to obtain floating images, respectively. MI curves are much peaked than $\mathrm{CC}$, especially with sinc and tri-linear interpolation. The voxel is isotropic with $1.4 \mathrm{~mm}$ on a side. Image data are the same used in Figure 1. 


\subsection{Rigid Body Registration Algorithm with Special Features}

The algorithm includes special features to improve robustness for registration of MR prostate images. ${ }^{1}$ Suppose the iMRI image slice is the reference image, the matching slice extracted from the high-resolution MRI volume is the floating image. We use a multi-resolution approach and perform registration from low to high resolution. We use $\mathrm{CC}$ at the two lower resolutions because it gives fewer local maxima and because it can be calculated faster than MI. We use MI at full resolution because of its peaked surface. To avoid local maxima, we include a restarting feature where registration is restarted with randomly perturbed parameters obtained from a uniform distribution about the initial transformation values at the current resolution being used. The algorithm restarts until the absolute $\mathrm{CC}$ is above a threshold of 0.5 as experimentally determined or the maximum number of restarts is reached. Absolute CC is used rather than MI because it has a well-defined range between 0 and 1 and because it provides an independent check of the MI result at the highest resolution. ${ }^{11}$

We record all important results following an optimization cycle including the $\mathrm{CC}$ and/or MI values and the transformation parameters. At the end of processing at a lower resolution, we always select the transformation parameters having the maximum CC value. We then scale the translation parameters appropriately and assign the new parameters to be initial values at the next higher resolution. At the highest resolution, MI instead of CC is the similarity measure, and we select the final transformation parameters to be those having the maximum MI value.

Additional algorithm details are now described. For registration, we use rigid body transformation (three translations and three rotations). We chose tri-linear interpolation instead of sinc because it is much faster and because it has comparable performance, as observed from Figures 1 and 2. For optimization, we use the downhill simplex method of Nelder and Mead. ${ }^{17}$ Optimization of similarity ends either when the maximum number of calculations is reached (typically 500) or the fractional change in the similarity function is smaller than a tolerance (typically 0.001). The input MRI volume is a 3D MR acquisition giving $256 \times 256 \times 128$ nearly isotropic voxels over a field of view covering the whole pelvis. We create isotropic voxels of about $1.4 \mathrm{~mm}$ on a side using 3D linear interpolation. We use IDL (Interactive Data Language, Research System Inc., Boulder, CO.) as the programming language. We use an initial guess assuming an identity transformation, i.e., all initial translation and rotation parameters are zero, because the patient is normally oriented approximately the same way from one scan to the next. We set the maximum numbers of restarts at 10,5 , and 3 , from low to high resolution, respectively.

\subsection{Simulation of iMRI Slice Images}

We used high-resolution MRI volumes to simulate iMRI images by creating thick slices and adding noise and receive coil inhomogeneity. ${ }^{11}$ Clinically, we typically use an iMRI slice thickness of $4.0-6.0 \mathrm{~mm}$. We averaged 3 slices 1.4 $\mathrm{mm}$ thick to create a $4.2 \mathrm{~mm}$ thick slice.

We added noise to the simulated iMRI image. MR noise is described by the Rician distribution, ${ }^{18}$ but at reasonably high signal values, the noise is accurately approximated with Gaussian white noise. ${ }^{19}$ We measured typical SNR on our open magnet system using a homogenous phantom and methods described elsewhere. ${ }^{20,21} \mathrm{We}$ then added Gaussian

noise to the simulated iMRI slice images either to match the measured SNR or to give much greater noise to further stress registration. We report noise experiments using the SNR of the simulated slice images. 


\subsection{Registration Evaluation}

Our standard evaluation method was to compare SV and VV registration. Since this relies on VV registration accuracy, we now review our previous results. ${ }^{1}$ For volume pairs acquired over a short time span from a supine subject with legs flat on the table, prostates were well aligned and prostate centroid displacements were typically $<1 \mathrm{~mm}$. The registration accuracy as determined from displacements of pelvic bony landmarks was $1.6 \pm 0.2 \mathrm{~mm}$. This error might be overestimated because it includes the uncertainty of locating the bony landmarks. The centroid error was slightly smaller because the prostate was at the volume center and rotation errors had less effect. From our success with VV prostate registration, we decided that we could obtain SV accuracy by comparing to VV registrations for those volume pairs having low VV registration error.

To compare SV and VV registration, we defined a rectangular volume of interest (VOI) just covering the prostate and calculated voxel displacements between the two registrations. To voxels within the VOI, we applied the transformations obtained by VV and by SV registrations. We then calculated the 3D displacements between the transformed voxels. The mean voxel distance was used as our metric of SV registration error. For the evaluation of algorithm robustness, we defined the SV registration as being successful when the 3D displacement was less than 2.0 $\mathrm{mm}$.

We used the multiple visualization features of RegViz, a program created in IDL in our laboratory, to visually evaluate registration results. First, we manually segmented prostate boundaries in image slices and copied them to corresponding slices from the other volume. This enabled visual determination of the overlap of prostate boundaries over the entire volume. We applied the same method to evaluate pelvic registration. Second, color overlay displays were used to evaluate overlap of structures. One image was rendered in gray and the other in the "hot - iron" color scheme available in IDL. To visualize potential differences, it was quite useful to interactively change the contribution of each image using the transparency scale. Third, we used a sector display, which divided the reference and registered images into rectangular sectors and created an output image by alternating sectors from the two input images. Even subtle shifts of edges could be clearly seen. ${ }^{1}$

\section{EXPERIMENTAL METHODS}

\subsection{Image Acquisition}

All MRI volumes were acquired using a 1.5 T Siemens MRI system (Magnetom Symphony, Siemens Medical Systems, Erlangen, Germany). An 8-element phased array body coil was used to ensure coverage of the prostate with a uniform sensitivity. Typically two anterior and two posterior elements were enabled for signal acquisition. We used two different MR sequences. First, we used a 3D RF spoiled gradient echo steady state pulse sequence (FLASH) with TR/TE/flip parameters of 12/5.0/60 which give 256 x 256 x 128 voxels over a 330 x 330 x 256-mm field of view (FOV) to yield $1.3 \times 1.3 \times 2.0-\mathrm{mm}$ voxels oriented to give the highest resolution for transverse slices. The acquisition time was $5.6 \mathrm{~min}$. This sequence was good for pelvic imaging but was not ideal for the prostate. Second, we used a 3D rapid gradient echo sequence (PSIF) designed to acquire the spin-echo component of the steady state response, rather then the free induction decay. The spin echo component formed immediately prior to the RF pulse, and it was shifted toward the prior RF pulse through appropriate gradient waveform design. The sequence with 9.4/5.0/60 (TR/TE/flip) yielded 160 x 256 x 128 voxels over a 219 x 350 x 192-mm rectangular FOV and 1.4 x 1.4 x 1.5-mm voxels oriented to give the highest resolution for transverse slices. There was over sampling at $31 \%$ in the slice direction to reduce aliasing 
artifacts. The acquisition time was 4.3 min. Most often, we used the second sequence, which gave excellent image contrast for the prostate and its surroundings.

\subsection{Imaging Experiments}

We acquired high-resolution MRI volumes from three volunteers. For each volunteer, three image volumes were obtained with an imaging session. Each volume was acquired with compatible conditions. Volunteers laid supine with legs flat similar to the position in routine MR scanning. Between volume acquisitions, volunteers got off the MRI table, stretched, and walked around to ensure that they would assume a different position when they laid back on the table. The coil array was centered on the prostate. All images of a volunteer were acquired with the same MRI acquisition parameters. In total, there are 9 pairs of high-resolution MRI volumes for registration.

\section{RESULTS AND DISCUSSION}

\subsection{Slice Orientation and Position}

As described in Section 2, we obtained relatively low noise, high-resolution MR images and simulated SV registration results. These data sets allowed us to test effects of noise and receive coil inhomogeneity in a controlled fashion. And, because we had substantial previous experience showing the accuracy of VV registration under comparable conditions, we could easily determine SV error by comparing results to VV registration.

We determined SV registration results for slices near the prostate in the three standard orthogonal orientations. Comparing to $\mathrm{VV}$, mean and standard deviation registration errors across 9 volume pairs and $45 \mathrm{SV}$ registration experiments were $0.4 \mathrm{~mm} \pm 0.2 \mathrm{~mm}, 0.5 \mathrm{~mm} \pm 0.2 \mathrm{~mm}$, and $2.6 \mathrm{~mm} \pm 1.6 \mathrm{~mm}$ for transverse, coronal and sagittal slices covering the prostate, respectively. Transverse slices worked best because they contain many relatively rigid anatomical structures. Coronal slices worked next best. Sagittal slices gave the largest error because they contained a large portion of the deformable bladder and rectum. We further found that transverse slices centered on the prostate produced better results than those above or below the prostate. Image slices above included the deformable bladder that could give an inconsistent structure from one volume to the next. Image slices below the prostate mainly contained muscle and fatty regions from the hips that could deform, again giving inconsistent image data.

\subsection{Noise Level}

The typical iMRI SNR under clinical conditions is about 25. Even when noise much exceeded this normal situation, registration results were quite good. A $100 \%$ success rate was achieved with an acceptance criterion of $<2.0 \mathrm{~mm}$ even when SNR was as bad as 10.

\subsection{Robustness}

The registration algorithm was quite robust for transverse slices covering the prostate. Using the nine volume pairs from three volunteers, the algorithm never failed for any transverse slice covering the prostate. In addition, the final registration result was insensitive to initial guesses within a large range, $[-60,+60] \mathrm{mm}$ for translations and $[-20,+20]$ degrees for rotations. With the restarting algorithm, we even successfully registered slices as much as $80 \mathrm{~mm}$ from the 
optimum. This working range is probably sufficient for clinical applications where we can ensure good starting values. Using the pelvic bones as markers and needle localization methods, ${ }^{22}$ we should be able to position the prostate within about $\pm 20 \mathrm{~mm}$. In addition, the patient normally lies supine in the MR bed with very little rotation $(< \pm 5$ degrees $)$.

\subsection{Comparison of Different Interpolation Methods}

Computation time and registration accuracy are two main factors to consider when choosing interpolation methods. Using tri-linear interpolation, the time for an SV registration was typically about $5 \mathrm{sec}$ on a Pentium IV, $1.8 \mathrm{GHz} \mathrm{CPU}$, with 1 Gbyte of memory. If the re-normalized sinc interpolation method was used, the time was $\sim 10$ min, a duration not acceptable for our application. The algorithm was written in IDL and could probably be made faster in a lower level language such as $\mathrm{C}$. We did not use nearest neighbor because of the obvious insufficient accuracy as induced from its flat peak of the similarity curves in Figure 2. A call to the Simplex optimization typically resulted in 50 to 105 similarity evaluations before the tolerance value $(0.001)$ was reached.

\subsection{Comparison of Three Alignment Techniques}

When registering iMRI image slices with high-resolution MR volumes, we compared three alignment techniques: (1) an iMRI thick slice to an MR thin slice, (2) an iMRI thick slice to the average of three MR thin slices, and (3) three copies of the iMRI thick slice to three MR thin slices. The second and third methods were tested because they were thought to better match the resolution of the high resolution volume to the the thick slide. There were no significant differences (voxel displacements $<0.1 \mathrm{~mm}$ ) among the three methods. Hence, we used the first method because of its simplicity.

\section{CONCLUSION}

The slice to volume registration algorithm is quite robust for transverse slice images covering the prostate. Since livetime iMRI images are used for guidance and registered images are used for adjunctive information, the registration accuracy is very probably adequate. It is quite feasible to include previously acquired high-resolution MRI or nuclear images into iMRI-guided treatment procedures. We are beginning to explore this application in animal experiments.

\section{ACKNOWLEDGEMENTS}

The algorithm developed in this research was supported by NIH grant R01-CA84433-01 to David L. Wilson and DOD grant DAMD17-02-1-0230 to Baowei Fei. Imaging techniques were developed under the support of NIH grant R33CA88144-01 to Jeffrey L. Duerk.

\section{REFERENCE}

1 B.W.Fei, A.Wheaton, Z.Lee, J.L.Duerk, and D.L.Wilson, "Automatic MR volume registration and its evaluation for the pelvis and prostate," Physics in Medicine and Biology, vol. 47, pp. 823-838, 2002.

2 M.V.Herk, A.Bruce, A.P.G.Kroes, T.Shouman, A.Touw, and J.V.Lebesque, "Quantification of organ motion during conformal radiotherapy of the prostate by three dimensional image registration," International Journal of Radiation Oncology Biology Physics, vol. 33, pp. 1311-1320, 1995. 
3 R.K.Tenhaken, J.D.Forman, D.K.Heimburger, A.Gerhardsson, D.L.Mcshan, C.Pereztamayo, S.L.Schoeppel, and A.S.Lichter, "Treatment planning issues related to prostate movement in response to differential filling of the rectum and bladder," International Journal of Radiation Oncology Biology Physics, vol. 20, pp. 1317-1324, 1991.

4 H.Gray. Anatomy, Descriptive and Surgical (The classic collector's edition). New York: Gramercy Books, pp. 1010, 1977.

5 B.Kim, J.L.Boes, P.H.Bland, T.L.Chenevert, and C.R.Meyer, "Motion correction in fMRI via registration of individual slices into an anatomical volume," Magnetic Resonance in Medicine, vol. 41, pp. 964-972, 1999.

6 Rohlfing, Torsten, West, Jay B., Beier, Jürgen, Liebig, Thomas, Taschner, Christian A., and Thomale, Ulrich Wilhelm. Registration of functional and anatomical MRI: Accuracy assessment and application in navigated neurosurgery. Computer Aided Surgery 5(6), 414-425. 2000.

7 T.S.Kim, M.Singh, W.Sungkarat, C.Zarow, and H.Chui, "Automatic registration of postmortem brain slices to MRI reference volume," IEEE Transactions on Nuclear Science, vol. 47, pp. 1607-1613, 2000.

8 J.Zhengping and P.H.Mowforth, "Mapping between MR brain images and voxel model," Medical Informatics, vol. 16, pp. 183-193, 1991.

9 B.W.Fei, A.Wheaton, Z.Lee, K.Nagano, J.L.Duerk, and D.L.Wilson, "Robust registration algorithm for interventional MRI guidance for thermal ablation of prostate cancer," Proceedings of SPIE Medical Imaging on Visualization, Display, and Image-Guided Procedures, vol. 4319, pp. 53-60, 2001.

10 B.W.Fei, J.L.Duerk, and D.L.Wilson, "Automatic 3D Registration for Interventional MRI-Guided Treatment of Prostate Cancer," Computer Aided Surgery, (In Press), 2003.

11 B.W.Fei, J.L.Duerk, D.T.Boll, J.S.Lewin, and D.L.Wilson, "Slice to volume registration and its potential application tointerventional MRI guided radiofrequency thermal ablation of prostate cancer," IEEE Transactions on Medical Imaging, (In Press), 2003.

12 B.W.Fei, D.T.Boll, J.L.Duerk, and D.L.Wilson, "Image registration for interventional MRI-guided minimally invasive treatment of prostate cancer,"Proceedings of the Second Joint EMBS/BMES Conference, Houston, TX, USA, October 23-26, pp. 1185, 2002.

13 F.Maes, A.Collignon, D.Vandermeulen, G.Marchal, and P.Suetens, "Multimodality image registration by maximization of mutual information," IEEE Transactions on Medical Imaging, vol. 16, pp. 187-198, 1997.

14 W.H.Press, S.A.Teukolsky, W.T.Vellerling, and B.P.Flannery. Numerical Recipes in C: The Art of Scientific Computing, Second Edition. New York: The Press Syndicate of the Cambridge University, 1992.

15 N.A.Thacker, A.Jackson, D.Moriarty, and E.Vokurka, "Improved quality of re-sliced MR images using renormalized sinc interpolation," Journal of Magnetic Resonance Imaging, vol. 10, pp. 582-588, 1999.

16 J.V.Hajnal, N.Saeed, E.J.SOAR, A.Oatridge, I.R.Young, and G.M.Bydder, "A registration and interpolation procedure for subvoxel matching of serially acquired mr-images," Journal of Computer Assisted Tomography, vol. 19, pp. 289-296, 1995.

17 J.Nelder and R.A.Mead, "A simplex method for function minimization," Computer Journal, vol. 7, pp. 308$313,1965$.

18 A.Macovski, "Noise in MRI," Magnetic Resonance in Medicine, vol. 36, pp. 494-497, 1996.

19 R.C.Gregg and R.D.Nowak, "Noise removal methods for high resolution MRI," IEEE Nuclear Science Symposium, vol. 2, pp. 1117-1121, 1997.

20 R.M.Henkelman, "Measurement of signal intensities in the presence of noise in MR mages," Medical Physics, vol. 12, pp. 232-233, 1985.

21 L.Kaufman, D.M.Kramer, L.E.CROOKS, and D.A.Ortendahl, "Measuring signal-to-noise ratios in MR imaging," Radiology, vol. 173, pp. 265-267, 1989.

22 J.S.Lewin, J.L.Duerk, V.R.Jain, C.A.Petersilge, C.P.Chao, and J.R.Haaga, "Needle localization in MR-guided biopsy and aspiration: Effects of field strength, sequence design, and magnetic field orientation," American Journal of Roentgenology, vol. 166, pp. 1337-1345, 1996. 
Baowei Fei, Kristin Frinkley, and David L. Wilson, "Registration algorithms for interventional MRI-guided treatment of the prostate", Robert L. Galloway, Editor, Proc. SPIE 5029, 192 (2003)

Copyright 2003 Society of Photo-Optical Instrumentation Engineers (SPIE). One print or electronic copy may be made for personal use only. Systematic reproduction and distribution, duplication of any material in this paper for a fee or for commercial purposes, or modification of the content of the paper are prohibited.

http://dx.doi.org/10.1117/12.479696 